\title{
Le néolibéralisme français ou comment penser le libéralisme au prisme des institutions
}

\section{Arnaud Diemer}

\section{(2) OpenEdition \\ 12 Journals}

Édition électronique

URL : http://journals.openedition.org/ei/5612

DOI : 10.4000/ei.5612

ISSN : 2553-1891

Éditeur

Association Économie et Institutions

Référence électronique

Arnaud Diemer, «Le néolibéralisme français ou comment penser le libéralisme au prisme des institutions », Économie et institutions [En ligne], 20-21 | 2014, mis en ligne le 01 juin 2014, consulté le 30 avril 2019. URL : http://journals.openedition.org/ei/5612 ; DOI : 10.4000/ei.5612

Ce document a été généré automatiquement le 30 avril 2019

Revue Économie et institutions 


\title{
Le néolibéralisme français ou comment penser le libéralisme au prisme des institutions
}

\author{
Arnaud Diemer
}

1 À la suite de la Grande dépression de 1929 et de la remise en cause du libéralisme, un certain nombre d'économistes d'inspiration libérale vont s'engager dans un vaste mouvement, visant d'une part, à faire renaître le libéralisme face à la poussée du collectivisme et du planisme (c'est le cas de Von Mises et de Friedrich Hayek) et d'autre part, à poser les bases d'une refondation du libéralisme (c'est la position de Walter Lippmann, Louis Rougier, Wilhelm Röpke...).

2 Ce mouvement n'est donc pas homogène. Louis Rougier ${ }^{1}$ en a bien conscience lorsqu'il rappelle que ce sont tout d'abord des idées dispersées qui esquissent vers la fin des années 30 « les linéaments d'une doctrine appelée par les uns, libéralisme constructeur, désignée par les autres néo-capitalismes et auquel l'usage semble prévaloir de donner le nom de néo-libéralisme» $(1939$, p. 9). Cependant le cœur de cette doctrine est constitué des deux positions suivantes : (1) dénoncer la montée du collectivisme et du planisme (la planisme autoritaire a rejeté l'organisation économique basé sur la concurrence en préconisant l'emploi d'une direction centralisée à toute l'économie) et (2) rejeter les thèses défendus par les tenants du laisser-fairisme (ce dernier a jeté les bases de la représentation du régime juridique du libéralisme - propriété privée et contrats - en idéalisant l'économie concurrentielle parfaite et en rejetant l'intervention de l'État).

Indéniablement, le colloque Lippmann, qui s'est déroulé à Paris (Institut international de coopération intellectuelle), du 26 au 30 août 1938, peut être présenté comme la première pierre de l'édifice (Cros, 1950; Denord, 2001; Augier, 2008), une esquisse d'institutionnalisation du libéralisme dans l'histoire des faits et des idées (Diemer, 2013). Ce colloque fût organisé par Louis Rougier ${ }^{2}$ à la suite de la parution de l'ouvrage de Walter Lippmann, The Principles of the Good Society (1937), qui aura un énorme retentissement sur le milieu intellectuel dans sa quête de liberté (Maurois, 1938). Traduit en français dès 1938, sous le titre La cité libre (éditions de Médicis), cet ouvrage se divise en deux parties 
(Clave, 2004). La première partie présente les théories et les actes du mouvement socialiste qui, dès 1870 , s'est efforcé d'instituer un ordre social dirigé ${ }^{4}$ : "Entre 1848 et 1870, le climat intellectuel de la société occidentale changea. L'ascension du collectivisme commença. L'Angleterre reste fidèle au libre-échange jusqu'à la guerre de 1914, mais partout ailleurs, la doctrine protectionniste croissait en popularité » (Lippmann, 1938, p. 55). La seconde partie cherche à comprendre pourquoi le développement de la pensée libérale s'est trouvé arrêté et pourquoi le libéralisme a perdu son influence sur le monde des affaires.

4 Les idées maîtresses de ce livre font écho à celles formulées au même moment par des auteurs tels que Jacques Rueff (La crise du capitalisme, 1935); Ludwig von Mises (Le socialisme , 1938) ; Lionel Robbins (L'économie planifiée et l'ordre international, 1938); Walter Lippmann (La Cité libre, 1938), Louis Rougier (Les mystiques économiques, 1938); Bernard Lavergne (Grandeur et déclin du capitalisme, 1938); Louis Marlio (Le sort du capitalisme, 1938). Le colloque Lippmann devait ainsi permettre aux différents protagonistes du colloque de se réunir en vue de « réviser le procès du capitalisme et de chercher à définir la doctrine, les conditions de sa réalisation, les tâches nouvelles d'un libéralisme véritable » (Rougier, 1939, ibid.). Pour Walter Lippmann, le colloque devait marquer le renouveau du libéralisme, c'est-à-dire rompre avec une philosophie héritée du $19^{\mathrm{e}}$ siècle. Une philosophie qui s'est révélée incapable de se perpétuer: "nous perdrions notre temps si nous nous imaginions que défendre la cause de la liberté équivaut à espérer que l'humanité revienne naïvement et sans réserve au libéralisme d'avant-guerre » [Lippmann W. (1938), p. 14]. La doctrine libérale devait donc être révisée, tout restait à écrire, mais pour cela, il convenait de s'attaquer au communisme, au national-socialisme et au fascisme, lesquels remettent en cause les fondements juridiques (la loi, la propriété privée, l'État) et moraux (l'équité, la justice) des sociétés occidentales.

5 Si le colloque Lippmann a donné une large place au sens à donner au mot libéralisme ${ }^{5}$, les débats ont montré que "penser le renouveau du libéralisme » n'était ni une question simple, ni l'expression d'un consensus. Au contraire, le colloque a révélé toute l'hétérogénéité de la pensée libérale (Nadeau, 2007, Denord, 2008). Les différents protagonistes prirent cependant le temps de proposer à la fin du colloque, une liste de problèmes théoriques et pratiques d'un retour au libéralisme, une manière d'harmoniser les positions de chacun et de fixer les futures orientations (création notamment du CIRL : Centre international de refondation du libéralisme).

6 Dans ce qui suit, nous ne chercherons pas à rendre compte de l'ensemble des idées véhiculées par ce vaste mouvement libéral. Nous avons pris le parti de nous focaliser uniquement sur les thèses défendues par le courant néolibéral français, et plus particulièrement les travaux de trois auteurs représentatifs de ce courant : Louis Rougier, Jacques Rueff et Maurice Allais.

7 Louis Rougier est avant tout un philosophe qui s'intéresse aux questions économiques. Son premier article date de 1920 et porte sur l'inflation monétaire. Il contribue par ailleurs au Bulletin quotidien de la Société d'Études et d'Informations Économiques (SEIF) que dirige son ami André François Poncet (Berndt, Marion, 2006, p. 43). Mais c'est surtout à partir d'une série d'ouvrages La Mystique démocratique (1929); La Mystique soviétique (1934); Les Mystiques politiques contemporaines (1935); Les Mystiques économiques (1938), d'articles et de conférences sur le libéralisme économique que Louis Rougier se fera connaître du milieu intellectuel anglo-saxon et français de l'entre-deux guerres ${ }^{6}$ (Denord, 2001, 2007 ; Diemer, 2011, 2013a). 
Jacques Rueff fût successivement Inspecteur des finances, Attaché financier de l'ambassade de France à Londres, Directeur adjoint du Mouvement général des fonds, Sous-gouverneur de la Banque de France, Conseiller économique du maréchal de Lattre de Tassigny, Président de l'Agence interalliée des réparations allemandes, Juge à la Cour de justice de la Communauté européenne du charbon et de l'acier, puis de la Communauté économique européenne (Margairaz, 2001 ; Frobert, 2010 ; Diemer, 2013). Son influence sur le mouvement néolibéral français fut essentielle (Lange, 1996 ; Arena, 2000).

Enfin, Maurice Allais n'a pas participé au colloque Lippmann, cependant ses lectures (compte rendu du colloque par Louis Rougier, 1939; les Mystiques politiques contemporaines et les Mystiques économiques) vont l'inciter à écrire à Louis Rougier en septembre 1945. Les préoccupations de Maurice Allais étaient alors purement économiques et libérales, sa vocation d'économiste (Allais, 2001, p. 332) avait été déterminée par les conséquences socio-économiques de la Grande dépression aux ÉtatsUnis (voyage en 1933) et le suivi des troubles sociaux en France (échec relatif du Front Populaire dans sa volonté d'engager des réformes de structures). Se sentant en "profonde sympathie avec les idées libérales exprimées dans les écrits [de Rougier]» (Allais, 1990, p.12), les deux hommes entament une intense correspondance de septembre 1945 à juillet 1947. À partir de cette date, ils prennent l'habitude de se rencontrer au sein du Groupe de Recherches Économiques et Sociales (GRECS) que Maurice Allais a fondé à la Libération avec Auguste Detœuf. Louis Rougier y fera deux conférences, l'une consacrée à la Réforme de la Constitution et la Sauvegarde des Libertés Fondamentales (novembre 1951), l'autre aux Causes du Développement de l'Occident (décembre 1960). Les 7 et 8 février 1859, Maurice Allais organise le Colloque pour une Société Libre au cours duquel Louis Rougier présente le Manifeste pour une Société Libre. Malgré les nombreux efforts des différents protagonistes - présence de Jacques Rueff et d'André François-Poncet - ce mouvement n'a qu'une expérience éphémère.

Le choix de ces trois auteurs repose sur les considérations suivantes. D'une part, ces auteurs s'inscrivent dans un processus de refondation du libéralisme (Louis Rougier en a été la cheville ouvrière; Jacques Rueff, un ardent défenseur et Maurice Allais; un ingénieur teinté d'un certain pragmatisme). D'autre part, tous les trois défendent l'idée que ce nouveau libéralisme suppose une ingérence (juridique) de l'État. Cette conception de l'État repose sur un compromis qu'il convient de délimiter avec précision. Enfin, leur positionnement les a amené à proposer un cadre théorique et pratique original, réconciliant les intérêts individuels et l'intérêt général, que ce soit le libéralisme constructeur de Rougier, le marché constitutionnel de Rueff ou la planification concurrentielle d'Allais. À travers ces trois auteurs, nous entendons montrer - à travers leurs positions communes mais également leurs antagonismes - que ce nouveau libéralisme a besoin d'institutions solides pour asseoir une certaine légitimité. Enfin, en guise de fil rouge, nous ferons référence aux travaux de Lionel Robbins (L'économie planifiée et ordre international, 1938) et ceux de Friedrich Von Hayek (notamment, la Route de la servitude, 1946), lesquels nous semblent assez bien représenter les débats de l'époque au sein du courant libéral : en l'occurrence, la question du plan et de sa pertinence dans les pays occidentaux ; le positionnement vis à vis du socialisme, du communisme et du planisme. 


\section{Des institutions pour faire face à la montée du collectivisme et au dogme du laisser-faire manchestérien}

11 S'il y a bien une thèse qui fait consensus au sein du mouvement libéral et du courant néolibéral français, c'est le bien le rejet du collectivisme (même si la position de Maurice Allais est moins tranchée que celle de ses homologues) et le refus du dogme du laisserfaire et du libéralisme manchestérien.

12 C'est tout du moins ce que semble penser Walter Lippmann dans les premières pages de son ouvrage, La Cité libre. Après 1870, les hommes se seraient ainsi mis à penser en termes d'organisation (concentrations industrielles, suppression de la concurrence), d'autorité et de pouvoir collectif (la conception de la démocratie change). À partir de 1917, certains États auraient même goûté à l'expérience du plan officiellement établi et le monde a l'occasion d'observer les expériences russe, italienne et allemande. La crise de 1929 associée à la débâcle du libéralisme (Dardot, Laval, 2007) ne fera que conforter les thèses collectivistes et totalitaires. C'est dans ce contexte qu'il convient de situer les origines du néolibéralisme ou plus généralement les tentatives de révision des idées libérales du $19^{\mathrm{e}}$ siècle. La recherche d'une troisième voie qui ne se confonde ni avec le planisme autoritaire, ni avec le laisser faire - laisser passer, est clairement évoquée par Louis Rougier dans son allocution au colloque Lippmann : «Être libéral, ce n'est pas, comme le "manchestérien", laisser les voitures circuler dans tous les sens suivant leur bon plaisir, d'où résulteraient des encombrements et des accidents incessants; ce n'est pas, comme le "planiste", fixer à chaque voiture son heure de sortie et son itinéraire; c'est imposer un Code de la route tout en admettant qu'il n'est pas forcément le même au temps des transports accélérés qu'au temps des diligences » (Rougier, 1939, p. 16).

Dans son ouvrage les Mystiques économiques (1938a), Rougier associe le collectivisme et le libéralisme manchestérien à des mystifications de l'esprit. Une doctrine devient mystique ${ }^{8}$ « lorsqu'on la soustrait au contrôle de l'expérience et à l'épreuve de la discussion, pour la traiter comme un dogme intangible, ou lorsqu'on fonde sur une base qui n'a aucun sens empirique ou rationnel et qui exprime seulement une conviction passionnée » (Rougier, La Mystique démocratique, 1929, p. 12). Par le terme mystique, Louis Rougier entendait ainsi dissocier la science économique, fondée sur l'expérience et la raison, et s'attachant à décrire les lois auxquelles obéissent les phénomènes économiques (lois qui régissent l'équilibre économique dans un régime de libre concurrence), des doctrines économiques proprement dites, lesquelles "explicitent simplement en les colorant de pseudosdémonstrations scientifiques, certaines attitudes mentales; en bref, certains partis pris passionnés » (1938a, p. 7). À l'image des valeurs religieuses d'autrefois, les idéologies économiques (et politiques) joueraient le rôle de croyances que l'on accepte aveuglément. L'économie dirigée, le corporatisme, le libéralisme manchestérien et le marxisme seraient des illustrations de ces mystiques.

Le renouveau du libéralisme ne peut donc passer que par un revirement de l'opinion publique et la démystification des pseudo-vérités. Cette nouvelle approche, appelée "libéralisme constructeur" ", doit rejeter à la fois l'anarchie du libéralisme manchestérien, le despotisme du planisme socialiste et du totalitarisme, et l'expérience du Front Populaire de $1936^{10}$ (Aron, 1937). Le libéralisme constructeur ne doit pas se 
confondre avec la théorie de l'école manchestérienne du laisser-faire - laisser passer (Cobden, 1835 ; Spencer, 1850) qui aboutirait à la suppression de la liberté par l'excès de liberté. En effet, si la concurrence est bien le fondement du système économique libéral, cette concurrence tend à disparaitre d'elle-même car elle entraîne le triomphe des plus forts et l'avènement du monopole (Piatier, 1938). Le libéralisme doit être progressif et constructif, il revendique l'autorité de la science économique (solution walrasienne à l'échange) quand il prétend que la libre concurrence permet d'assurer le maximum de satisfaction; il implique tout un système de lois sur les contrats, les sociétés anonymes, les ententes patronales, les syndicats ouvriers, les garanties professionnelles, l'héritage, le statut de la propriété... de manière à ce que les monopoles, les holdings, le pouvoir des syndicats... ne puissent voir le jour : «Ce que j'appelle libéralisme constructif n'est pas identifiable à la théorie manchestérienne du laisser-faire, laisser passer, car un tel libéralisme se détruit lui-même en aboutissant, par le seul jeu de la concurrence et de la sélection naturelle, à une économie de monopole correspondant à un régime ploutocratique. Le libéralisme constructif implique un ordre juridique positif tel que la possibilité de la libre concurrence soit toujours sauvegardée, c'est-à-dire un ordre juridique tel que la formation des trusts, des holdings, etc. devienne impossible, aussi bien du reste que serait impossible la tyrannie syndicale, imposant des conditions de salaires et d'embauche contraire à l'équilibre du marché du travail» (Rougier, 1938a, p. 34).

15 Cette recherche d'une troisième voie est également bien présente dans le courant ordolibéral allemand incarné par Wilhelm Röpke ${ }^{11}$ (1940) et les travaux de Lionel Robbins concernant l'économie planifiée. Dans son ouvrage Explications économiques du monde moderne, Röpke (1940) part du constat que l'économie politique aurait failli à sa tâche, à savoir, l'explication de la réalité et de ses problèmes. Cette responsabilité incombe aux économistes qui n'ont pas toujours su rendre accessible leur science. Pour comprendre la société dans son ensemble, et la crise qu'elle traverse, l'économiste ne doit pas se soucier exclusivement des problèmes économiques, mais aussi des fondements juridiques, sociologiques, anthropologiques, politiques, moraux et théologiques de la société. En effet, la crise de 1929 a montré les limites et les insuffisances du laisser-fairisme (urbanisation non maitrisée, monopolisation, réglementation excessive, domination des intérêts de classes...). La liberté économique est une condition nécessaire mais pas suffisante de la "cité libre», elle doit être rattachée à un état social dans lequel les individus mènent une vie fondée sur la propriété privée, une vie qui assure l'indépendance intérieure et extérieure. Afin de faire face à la montée du collectivisme, Wilhelm Röpke propose ainsi une troisième voie, une solution qui doit permettre "d'édifier un ordre économique et social fondé sur la liberté, la justice et la dignité humaine, tenant compte de la nature humaine, un ordre harmonieux, bien équilibré, qui, mettant en mouvement le plus fort de tous les instincts, l'instinct de conservation, ajoute encore aux autres avantages celui d'un bon rendement matériel» (1940, p. 19). De son côté, Lionel Robbins s'est appuyé dans son ouvrage L'économie planifiée et l'ordre international (1938), sur une étude documenté des plans utilisés dans les économies occidentales et orientales à la suite de la crise de 1929. Il note que si le planisme national mène au gaspillage et à l'insécurité et que le planisme international de certaines branches du commerce et de l'industrie n'améliore pas la situation, la solution pourrait prendre la forme d'un planisme libéral. En effet, contrairement à ce que l'on a coutume d'admettre, les concepts de plan et de libéralisme ne sont pas antinomiques. L'essence d'un plan, c'est qu'il constitue une tentative d'adaptation des moyens aux fins : « dans un monde soumis 
aux changements, l'essence et la condition de succès d'un plan organisant la production, c'est qu'il fournisse une continuelle adaptation aux variations des conditions techniques et des demandes des consommateurs » (1938, p. 201). Or, le libéralisme international se présente généralement « comme un ensemble d'institutions spécialement désignées pour faire face aux difficultés de l'organisation économique sur une échelle internationale » ( ibid.). Le gouvernement et les organismes d'État joueraient ainsi un rôle des plus importants et des plus indispensables dans le plan libéral.

16 Selon Lionel Robbins, c'est faute d'avoir perçu cette coopération que les libéraux du XIX siècle ont dénié toute fonction au gouvernement et bâti leur philosophie sur un ensemble de croyances naïves. En effet, si le marché libre et la propriété privée sont les symboles du libéralisme, ils n'existent et ne sont maintenus que dans le cadre des institutions. En somme, ni la propriété, ni les contrats ne sont naturels et spontanés, ils sont essentiellement la création de lois complexes ${ }^{12}:$ "L'idée d'une coordination des activités humaines au moyen d'un système de règles impersonnelles, à l'intérieur duquel les relations spontanées qui se produisent conduisent au bénéfice mutuel, est une conception au moins aussi subtile, au moins aussi ambitieuse que celle qui consiste à faire prescrire de façon positive chaque action ou chaque catégorie d'actions par une autorité planiste centrale : et elle n'est peut-être pas en moindre harmonie avec les besoins d'une société spirituellement saine. Nous pouvons blâmer les enthousiastes qui, dans leur intérêt trop poussé à l'égard de ce qui se passait sur le marché, n'ont pas accordé assez d'attention à son armature indispensable. Mais que dirons-nous de ceux qui discutent perpétuellement comme si cette armature n'existait pas ? » (1938, p. 204).

17 Robbins ne s'arrête cependant pas là, il reconnaît que le maintien du système libéral n'épuise pas les attributions de l'État. Le système de marché comporte en effet des limites. D'un côté, il existe des besoins qui ne peuvent être satisfaits que de façon collective (exemple des services sociaux, des procédures de vaccination à grande échelle). De l'autre, certains besoins formulés individuellement, ne peuvent pas engendrer une réponse privée et spontanée (exemple des voies de communications).

Ainsi, il serait faux de considérer que les propositions du libéralisme excluent toute forme de plan et que ce sont les institutions libérales qui seraient à l'origine du chaos laissé par la crise de 1929. Le libéralisme serait un plan qui n'a encore jamais eu la chance de s'exercer. Les corporations, les monopoles, les tarifs protecteurs... n'ont pas permis au planisme libéral de s'exprimer totalement. Robbins en appelle ici à l'histoire. De 1840 à 1870, le courant libéral s'est répandu dans toute l'Europe (diminution des tarifs douaniers, liberté individuelle, entreprises indépendantes, division internationale $\mathrm{du}$ travail, Traité du commerce franco-anglais de 1860...) sans pour autant parvenir à y prendre racine. Robbins avance trois explications susceptibles d'expliquer cet échec. Dans un premier temps, le modèle libéral anglais s'est heurté au modèle de l'État social allemand. L'échec de 1848 et l'unification de l'Allemagne par les armes et le sang vont créer toutes les conditions d'un retour aux principes mercantilistes. Le système de List et Schmoller se substitue à celui de Smith et Ricardo. En 1880, Bismarck impose de lourdes taxes sur le fer et l'acier. Dans un deuxième temps, les efforts du socialisme visant à proposer une organisation économique différente du modèle de la libre entreprise, donnent des résultats mitigés. Le socialisme utopique de Saint-Simon, d'Owen, de Fourier, de Cabet qui renvoie directement au mouvement coopératif et syndicaliste, ainsi qu'aux nombreuses expériences communautaires (les fameux phalanstères de Fourier ou les usines modèles d'Owen) décline à partir de 1870. Il est supplanté par le marxisme qui 
s'impose comme l'idéologie majeure du socialisme: "Avec le déclin des utopistes français, le socialisme retomba dans le mysticisme messianique du déterminisme marxiste - ou s'allia aux intérêts spéciaux du restrictionnisme syndicaliste. Sa propagande, bien que se disant internationale, eut pour effet d'affaiblir la confiance dans la liberté du marché et de fortifier le mouvement vers la restauration des contrôles internationaux » (1938, p. 210). Dans un troisième temps, les premiers libéraux n'ont pas cerné les véritables enjeux et la portée de leurs réformes. L'harmonie des intérêts individuels ne pouvait pas se satisfaire d'un cadre national. Tôt ou tard, les politiques suivies laissaient souvent la place aux intérêts particuliers et aux retours des privilèges. La réaction nationale (notamment l'arrivée au pouvoir d'Hitler) a joué un rôle important dans l'arrêt de l'extension du libéralisme. Ainsi, depuis 1870, la sphère internationale serait sous l'influence de la pensée allemande et de la politique allemande. Le planisme libéral n'a jamais pu être mise en place, écrasé qu'il était par la réaction de forces contraires : "Il est difficile d'exagérer l'influence exercée par l'existence, au centre de la civilisation européenne, d'une puissance dont les dirigeants et les penseurs rejetaient ouvertement le libéralisme et considéraient les idéaux ataviques de l'impérialisme comme le premier et le dernier mot de toute politique » (1938, p. 211).

Finalement Lionel Robbins prône la nécessité d'instaurer un libéralisme international. Pour ce faire, il convient de définir un cadre légal approprié au fonctionnement des marchés et au maintien de la propriété privé. Le gouvernement devra participer à l'édification de ce cadre et suppléer aux principes de la propriété privée lorsque ces derniers sont inapplicables ou attaqués. Le renouveau du libéralisme passe également par une exposition juste et complète de ses avantages et limites. D'une part, face à une société nécessairement imparfaite (distribution des richesses), on ne peut espérer constituer un système pur. Une autre manière de rappeler qu'il serait «faux de revendiquer, pour le système libéral, une perpétuelle réussite de l'équilibre parfait de la concurrence telle que l'énonce la théorie pure» (1938, p. 229). À défaut d'une perfection des calculs économiques qu'offrent les modèles abstraits, il convient de rechercher les institutions qui permettent à la concurrence de s'exprimer dans la pratique. D'autre part, le libéralisme n'est pas exempt d'erreurs. L'acte de production repose sur l'anticipation de la demande. Or rien ne nous garantit que les anticipations des producteurs seront autoréalisées. Ces dernières peuvent être imparfaites et amener le marché à sanctionner les plans défectueux: "C'est un trait essentiel du système libéral que les dirigeants de l'industrie ne sont pas à l'abri des conséquences de leurs propres erreurs. Les organismes gouvernementaux ont leurs fonctions propres. Ce n'est pas le rôle de ces fonctions d'empêcher les pressions caractéristiques du marché de se produire » (1938, p. 230). Dans certains cas, l'erreur collective prendra la forme d'un effet de contagion et conduira à des errements importants. Selon Robbins, il conviendra de développer les institutions de marché capables d'atténuer ces fluctuations.

Les travaux de Lionel Robbins ont eu une certaine influence sur ceux de Maurice Allais ${ }^{13}$ (1946, 1947, 1949, 1950). Cherchant à dépasser l'opposition systématique entre les partisans de l'organisation libérale manchestérienne et ceux de la planification centralisée, Maurice Allais considérait que le planisme concurrentiel pouvait conjuguer à la fois «les avantages fondamentaux d'une économie de marché et ceux d'une action consciente de l'État suivant un Plan en vue de la réalisation d'une économie à la fois plus efficace et plus juste » (Allais, 1947a, p. 1). Par l'intermédiaire du planisme concurrentiel, Allais entendait ainsi poser les bases normatives et positives du néolibéralisme français 
en analysant précisément l'interdépendance entre deux institutions, le marché et l'État (Diemer, 2010, 2012). Le "laisser-fairisme» manchestérien, rappelle Maurice Allais (1945b), a conduit les libéraux à la conception d'un monde imaginaire de concurrence parfaite dans lequel les problèmes posés par la production, la répartition se trouvaient résolus. Ce «laisser-fairisme » aurait introduit deux biais : la représentation du régime juridique de la propriété et des contrats, régis par un droit naturel révélé par la Providence; l'idéalisation d'une économie concurrentielle parfaite et le rejet de l'ingérence de l'État. La doctrine totalitaire repose quant à elle sur l'idée qu'il existerait une autorité centrale capable de planifier, d'organiser et de diriger de manière efficace une économie complexe (Lange, 1949).

La planification concurrentielle aboutit ainsi à l'affirmation suivante: si l'intérêt individuel, guidé par la liberté économique, constitue bien un moteur, il ne faut pas que cette liberté dégénère en anarchie. Il convient de l'organiser par la loi dans un cadre juridique qui satisfasse l'intérêt général : «Essentiellement bienfaisante, la concurrence est possible, mais elle n'est pas spontanée, ni automatique et elle ne peut nécessairement exister qu'organisée dans le cadre de la loi » (Allais, 1946c, p.1). Cette inspiration très institutionnaliste - que n'aurait pas réprouvée Léon Walras (1909) - fait de Maurice Allais un partisan de l'économie concurrentielle organisée. La condition essentielle de la liberté économique, c'est l'autorité toute puissante de l'État, notamment pour supprimer les profits des monopoles et les rentes de rareté (terre).

Sur le plan des idées, la planification concurrentielle constituait un triple enjeu.

- elle permettait à Maurice Allais de dissocier les économies à planification centrale des économies comportant un plan. Par cette distinction, Maurice Allais entendait faire du Plan, une " idée force ", dans la diffusion de ses travaux, mais également rappeler que le planisme continuait à gagner du terrain (Myrdal, 1960 ; Shonfield, 1967). Au lendemain de la $2^{\text {nde }}$ guerre mondiale, Maurice Allais n'hésitait pas à attribuer l'inefficience de l'économie française à son organisation institutionnelle, planificatrice et autoritaire: «Le second facteur qui, à notre avis, permet d'expliquer pour une grande part la supériorité d'efficience actuelle des États-Unis, c'est l'organisation concurrentielle à base de prix de marché extrêmement favorable à la production, qui caractérise l'économie américaine alors l'économie française étouffe dans bien des secteurs, dans le carcan d'une planification centrale et autoritaire dont les méfaits ne devront jamais être dénoncés avec trop de force " (Allais, 1949f, p.191). Le capitalisme français, associé à partir de 1946 au système d'interventions et d'incitations sélectives de hauts commissaires (Commissariat au Plan), était selon lui incompatible avec les principes de l'organisation concurrentielle et du Traité de Rome (1958): «Si dans son principe fondamental, la planification française dite indicative, correspond assez bien à ce que pourrait être une politique cohérente s'efforçant de promouvoir une situation d'efficacité maximum, elle montre dans ses déviations quelle distance il peut y avoir entre un principe déclaré et ses applications... Dans son principe, la planification française repose essentiellement sur la conjugaison de deux éléments: le fonctionnement d'une économie de marchés et la diffusion d'une large information concernant l'évolution probable de l'économie, compte tenu des déclarations du gouvernement concernant sa propre sphère. Dans son principe, elle laisse entièrement au marché le soin de résoudre les conditions correspondant à une situation d'efficacité maximum... Malheureusement sur le plan des applications... la politique française et le plan lui-même présentent d'innombrables déviations. Partout les autorités publiques multiplient leurs interventions sélectives et discrétionnaires » (Allais, 1967, p. 89-91). Dans les années 
60, période phare de l'ingénieur économiste, la planification française rechercha « une voie moyenne conciliant l'attachement à la liberté et à l'initiative individuelles avec une orientation commune du développement » (Massé, 1965, p. 144). Il s'agissait à la fois «de préserver l'économie de marché de déviations souvent tentantes » et « d'écarter la tentation dirigiste et ses effets pervers " (Massé, 2002, p. 139). La notion de planification indicative fut ainsi progressivement abandonnée au profit de celle de programmation. La planification mettait ainsi davantage l'accent sur la notion de prospective économique. Le modèle français pouvait dès lors réunir une philosophie inspirée du principe de l'économie de marché et des dispositions inspirées de l'économie du Plan, une forme d'institutionnalisme à la Française.

- elle trouvait un terrain d'application dans développement des pays sous-développés. Son ouvrage Le Tiers Monde au Carrefour: Centralisation autoritaire ou planification concurrentielle (1961) devait à la fois mettre en doute les affirmations dogmatiques de la planification centralisée et engager les pays sous-développés sur la voie d'un certain libéralisme. Une économie libérale suppose: (i) un ordre politique, ce dernier implique des institutions politiques suffisamment stables et l'existence d'élites politiques et administratives compétentes; (ii) un cadre politique et social ouvert dans lequel tous les individus puissent s'épanouir; (iii) un système de lois concernant la fiscalité, les conditions de travail, l'enseignement... qui conditionne toute l'efficacité d'un système économique ; (iv) un plan monétaire susceptible d'assurer l'expansion dans le plein emploi et l'équilibre de la balance des paiements; (v) une action directe et importante de l'État partout où elle est irremplaçable.

- Enfin, elle permettait à Maurice Allais de se désolidariser de la position très dogmatique de Friedrich Hayek (son refus catégorique de toute propriété collectiviste et de toute intervention de l'État). Ayant participé en avril 1947, aux côtés notamment de Milton Friedman, Ludwig von Mises, Friedrich Hayek, Franck Knight, Lionel Robbins, George Stigler à la réunion de la société du Mont Pèlerin, Maurice Allais refusa de signer le texte constitutif. Il motiva ce refus en rappelant qu'il avait toujours été partisan de la propriété collective toutes les fois où la structure économique d'un secteur ne pouvait pas se prêter à la concurrence (Allais, 1946d, 1947d, 1948c).

Ce dernier point est important à souligner et nous amène à revenir sur la position de Friedrich Hayek. D'une certaine manière, l'ouvrage de Maurice Allais, Abondance et Misère (1946), peut être interprété comme une réponse à celui de Hayek, The Road of Freedom (1943), traduit en français sous le titre, la Route de la servitude (1946). Précisons pour la petite histoire que les deux ouvrages ont été édités par la Librairie Médicis.

Dans son ouvrage, Hayek entendait rappeler que nous avions abandonné peu à peu l'idée de liberté économique (Dickinson, 1940) pour aller vers le socialisme, une nouvelle forme d'esclavage en rupture brutale avec toute forme d'évolution de la civilisation: "Nous abandonnons rapidement, non seulement les idées de Cobden et de Bright, d'Adam Smith et de Hume, ou même de Locke et de Milton, mais encore une des caractéristiques les plus saillantes de la civilisation occidentale telle qu'elle s'est édifiée sur les fondations posées par le christianisme, par la Grèce et par Rome » (1946, p. 17). Cette situation serait en partie le résultat d'une confusion entre individualisme et égoïsme. C'est tout du moins le fil rouge que tente de donner Hayek à ses lecteurs : «Ce n'est que petit à petit qu'il nous sera possible au cours de cet ouvrage d'expliquer le contraste entre les deux principes » (1946, p. 18). Les principes du libéralisme seront examinés dans le chapitre 1 intitulé La route abandonnée. Hayek insiste sur le insiste sur le fait qu'il n'y a rien dans les principes 
du libéralisme qui permette d'en faire un dogme immuable. Il n'y a pas de règles stables, fixées une fois pour toutes. Il y a juste un principe fondamental, à savoir que dans la conduite des affaires, il convient de faire le plus grand usage possible des forces sociales spontanées et de recourir le moins possible à la coercition. Hayek en conclut que « Rien n'a sans doute tant nui à la cause libérale que l'insistance butée de certains libéraux sur certains principes massifs, comme avant tout la règle du laissez-faire» $(1946$, p. 20). Contrairement à ses homologues français, Hayek ne condamne cependant pas le laisserfairisme. Selon lui, un tel positionnement était même " nécessaire et inévitable » (ibid.). Nécessaire car seule une règle rigide pouvait être efficace (l'existence d'intérêts particuliers jouissant de bénéfice immédiats aurait causé d'immenses préjudices indirects). Inévitable car la position du laissez-fairiste aurait pu être percée à jour à tout moment (l'existence d'exceptions étant susceptible d'ébranler toute structure, même la plus pertinente). Hayek finit par en conclure qu'une politique qui proposerait une amélioration progressive du cadre des institutions afin qu'il soit compatible avec une société libre, peut s'avérer contre-productive, voire inefficace si un tel changement prenait du temps ou se caractérisait par un certain attentisme : « Il y a, en particulier, une immense différence entre créer délibérément un système où la concurrence jouera le rôle le plus bienfaisant possible, et accepter passivement les institutions telles quelles sont » ( ibid.). En d'autres termes, les progrès de la politique libérale sont conditionnés par notre compréhension des forces sociales et des conditions les plus favorables à leur fonctionnement: "L'attitude d'un libéral à l'égard de la société est comme celle d'un jardinier qui cultive une plante, et qui, pour créer les conditions les plus favorables à sa croissance, doit connaître le mieux possible sa structure et ses fonctions » (ibid.).

Si Hayek attribue l'abandon des principes essentiels du libéralisme à la lenteur des progrès de la politique sociale, aux comportements de ceux qui utilisaient le libéralisme pour défendre des privilèges anti-sociaux et à l'ambition illimitée portée vers les améliorations matérielles, il note qu'une telle situation ne serait pas arrivée sans une forme d'utopie portée par le projet socialiste. En effet, pour parvenir à ses fins, le socialisme a eu recours à la promesse d'une nouvelle liberté, qui n'était rien d'autre que la très ancienne exigence d'une égale répartition des richesses : « Il est hors de doute que la promesse d'une plus grande liberté est devenue une des armes les plus efficaces de la propagande socialiste et que les gens croient authentiquement et sincèrement que le socialisme apporte la liberté. La tragédie est d'autant plus atroce s'il est prouvé que la route de la liberté promise ne mène qu'à la servitude. C'est incontestablement cette promesse de liberté qui a entraîné un nombre de plus en plus grand de libéraux sur la voie socialiste, qui leur a masqué le conflit qui existe entre les principes fondamentaux $d u$ socialisme et ceux du libéralisme et qui a souvent permis aux socialistes d'usurper jusqu'au nom du vieux parti de la liberté » (1946, p. 26).

Mais la critique de Hayek ne s'arrête pas aux frontières de l'idéologie socialiste, elle est encore plus virulente lorsqu'il s'agit d'évoquer sa méthode particulière, la planification centralisée : «Dans cette acceptation, le mot socialisme signifie abolition de l'entreprise privée, de la propriété privée des moyens de production, et création d'un système «d'économie planifiée » où le chef d'entreprise travaillant pour un profit est remplacé par un organisme central» (1946, p. 30). La controverse sur le socialisme devient ainsi une controverse sur les moyens, et la situation se complexifie, selon Hayek, lorsque le planisme économique (outil de la réforme socialiste) peut être utilisé dans d'autres circonstances. Ainsi, un plan libéral dans lequel il suffirait de tracer un cadre permanent 
et rationnel à l'intérieur duquel les individus se livreraient à leurs activités, conformément à leurs plans personnels, n'en constitue pas un aux yeux des planistes socialistes. Pour parvenir à atteindre un état idéal de la distribution des richesses, ces derniers n'entrevoient le plan que sous la forme d'une direction centralisée de toute l'activité économique.

La condamnation hayekienne du socialisme et de sa méthode (l'économie planifiée) contraste avec l'ambition de Maurice Allais (1949c) de faire de la planification concurrentielle, un véritable projet visant à concilier et à harmoniser les aspirations du libéralisme et du socialisme les principes du libéralisme et la justice sociale inhérente au marxisme: "Nous pensons quant à nous que [l'organisation économique et sociale] empruntera au libéralisme sa technique extraordinairement efficace de gestion décentralisée à base de prix concurrentiels et au marxisme son haut souci de justice sociale » (Allais, 1949c, p. 1). Dans cette volonté de créer un pont entre le libéralisme et le socialisme, Maurice Allais ira jusqu'à proposer de remplacer le concept de planification concurrentielle par celui de «socialisme concurrentiel» (Allais, 1947e) et de promotion des élites de gauche : "Nous sommes convaincus que l'idée concurrentielle a un immense avenir, mais notre conviction, chaque jour plus grande, est que seuls peuvent réussir à la mettre efficacement en œuvre les hommes dont les aspirations sociales sont dans leurs fondements mêmes celles des partis de gauche » (Allais, 1949c, p. 7).

Toutefois, la condamnation du socialisme n'est pas l'unique trait d'opposition entre Hayek et Allais, l'idée même de plan est inacceptable aux yeux de Hayek.

\section{Penser les institutions dans un projet de rénovation du libéralisme}

Les efforts de rénovation du libéralisme, initiés par Louis Rougier, Jacques Rueff et Maurice Allais, ne se sont pas contentés de proposer une alternative au laisser-fairisme et au planisme autoritaire, ils ont également permis de penser les institutions (notamment l'importance des droits de propriété, le rôle des lois et des règles du jeu du marché, ainsi que celui de l'État) dans un projet d'inspiration libéral. La notion d'institution est ici comprise dans son acceptation la plus large, "c'est l'ensemble des règles de droit que l'organisme institué a mission de mettre en œuvre» (Rueff, 1958, p. 2), cependant elle donne aux thèses des néo-libéraux un certain réalisme à leur conceptualisation du système économique.

Du point de vue de l'histoire de la pensée économique, cette posture du libéralisme n'est pas nouvelle. Dans son Cours complet, J-B Say (1840) consacre la quatrième partie de l'ouvrage à l'influence des Institutions sur l'économie des sociétés. L'auteur nous propose ici une vision très large des institutions, il s'agit bien entendu des lois et des conventions, mais également de toutes les institutions sociales qui favorisent la liberté d'entreprendre et la production de richesses (Diemer, Laperche, 2014). À ce titre, la division du travail peut être érigée en véritable institution sociale. L'accroissement des utilités produites qu'elle permet est dû principalement à la possibilité de conclure des échanges. Or ces échanges ne sont possibles que lorsque les hommes vivent dans des sociétés nombreuses. Ces dernières sont des lieux où les connaissances nouvelles s'ajoutent aux connaissances anciennes, où les arts se perfectionnent et les sciences se transmettent à l'ensemble des individus: "L'agglomération des hommes n'est pas moins nécessaire pour que les 
connaissances utiles se conservent et s'accroissent. Les observations, l'expérience d'un homme se perdraient aisément, s'il n'était entouré de beaucoup d'autres qui peuvent les recueillir et les transmettre à beaucoup d'autres" (1840, [2007, p. 510). Toutes ces institutions ne doivent servir qu'un unique objectif: satisfaire les besoins de la Nation. C'est pourquoi Say se propose d'appréhender les institutions qui sont favorables ou contraire au bien être des sociétés, des institutions qui participent de près ou de loin à la libéralisation des forces productives.

31 La première de ces institutions, c'est le droit de propriété. On retrouve ici l'approche institutionnelle à minima des libéraux français et anglais. Le droit de propriété est « la faculté exclusive garantie à un homme, à une association d'hommes, de disposer à leur fantaisie de ce qui leur appartient » (1840, [2007, p. 521]). Si la philosophie recherche les fondements du droit de propriété, si le droit établit les règles de transmission des choses possédées et si la science politique s'attelle à chercher les plus sûres garanties de ce droit, l'économie politique considère quant à elle que le droit de propriété est un fait. Sans lui, point de richesses, étant donné que ces dernières reposent sur l'évaluation des choses que l'on possède : «Je vous ferais observer que la propriété n'est un fait, qu'autant qu'elle est réelle. Une propriété qui serait illusoire n'aurait point les effets d'une propriété réelle. Elle n'est un fait que lorsqu'elle ne gît pas uniquement dans de vaines déclarations, lorsque les lois qui l'établissent ne sont pas mal faites ou mal exécutées, lorsqu'elles ne sont pas impunément violées; violées par d'autres lois, ou par les dépositaires mêmes de l'autorité à qui l'exécution en est confiée » (ibid.).

Le droit de propriété devient ainsi le garant de la libéralisation des forces productives toutes les fois qu'il attribue la propriété du produit au propriétaire des fonds productifs auxquels ce produit est associé. De ce fait, la propriété du fond et du produit issu de ce fond sont les principaux déterminants de la valeur et de l'accumulation de la richesse. Si l'on est amené à remettre en cause l'une de ces deux propriétés et notamment la seconde, aucun propriétaire de fond ne cherchera à le mettre en valeur et toute accumulation sera purement illusoire. Ce dernier point permet de comprendre les conséquences de la libéralisation des forces productives via le respect du droit de propriété, il est possible, d'une part, de donner une valeur aux choses (notamment des choses qui se transmettent par le marché et grâce à la division du travail) et d'autre part, d'accumuler des richesses. Le droit de propriété exclusif ouvre ainsi la porte à l'accumulation illimitée des richesses.

Une autre illustration de la place des institutions dans le corpus théorique libéral concerne l'articulation marché - concurrence - État. On la retrouve notamment dans les travaux de Léon Walras ${ }^{14}$. Dans les Éléments d'économie politique pure (1874), Walras précise que la supériorité de la concurrence pure ne prouve pas celle du laisser-faire, laisser passer (Diemer, Lallement, 2004). Certes, il reconnaît avoir montré que "la liberté procure, dans certaines limites, le maximum d'utilité » (Walras, 1874, p. 335). Mais, contre les libéraux qui condamnent l'intervention de l'État, il affirme très clairement la nécessité de cette intervention pour organiser les marchés concurrentiels. "Dire libre concurrence n'est pas du tout (...) dire absence de toute intervention de l'État. Et, d'abord, cette intervention est nécessaire pour établir et maintenir la libre concurrence là où elle est possible» (Walras, 1898, p. 475). Pour Walras, la concurrence n'est pas une situation spontanée de l'économie. La « force » de la concurrence, qui imposera aux échangeurs des prix, devra cependant être encadrée par une autre force. Il appartient ainsi à l'État d'organiser la concurrence (Potier, 1999) : «La partie économique de la science sociale comprend en réalité trois sciences distinctes et par leur objet et par leur caractère : 1 . 
l'économie politique pure ou l'étude des lois en quelque sorte naturelles et nécessaires, suivant lesquelles l'échange, la production, la capitalisation et la circulation de la richesse sociale tendraient à se faire sous un régime hypothétique de libre concurrence organisée (ce qui est tout autre chose que le simple laisser-faire); 2. l'économie sociale ou la recherche des lois morales qui devraient présider à sa répartition; et 3. l'économie politique appliquée ou la recherche des règles d'utilité suivant lesquelles devrait s'effectuer sa production. Or, j'estimais que, par le défaut de la première de ces trois sciences, le deux autres n'existaient pas» $\left(1874\right.$, vol II, p. 507) ${ }^{15}$. Comme les lois civiles devaient encadrer les lois naturelles afin de garantir le principe de propriété (Hume ${ }^{16}$, 1740), l'État et la législation ont pour fonction de garantir la libre concurrence: «Instituer et maintenir la libre concurrence économique dans une société est une œuvre de législation, et de législation très compliquée, qui appartient à l'État " (Walras, 1898, vol. X, p. 427).

Droit de propriété, concurrence organisée et intervention de l'État constituent au sein du courant néolibéral français des formes institutionnelles susceptibles de garantir une certaine forme de libéralisme. En s'inscrivant dans le prolongement des travaux de Say et Walras, Louis Rougier, Jacques Rueff et Maurice Allais réitèrent l'idée qu'il existerait une école française teinté de libéralisme mais également profondément ancrée dans une certaine forme d'interventionnisme de l'État.

Le libéralisme constructeur de Rougier (1938) suppose ainsi une ingérence juridique de l'État (Steiner, 2005), thèse qui rejoint les positions de Jacques Rueff, de Maurice Allais et celles de l'ordolibéralisme allemand prôné par Wilhelm Röpke ${ }^{17}$ (Bilger, 1966). La propriété, les contrats, les différentes sortes de sociétés ${ }^{18}$, la monnaie, l'organisation bancaire sont en effet des créations de la loi. Ces faits économiques n'existent que par un ensemble de droits, de garanties et d'obligations sanctionnés par l'autorité de l'État. Dès lors, l'État libéral n'a pas pour fonction de créer des équilibres artificiels, mais bien de rétablir les équilibres naturels entre épargne et investissement, entre production et consommation: "Ce que veut le libéralisme constructeur, c'est, au sein d'un régime contractuel que la loi peut toujours modifier et assouplir, assurer l'adaptation de la production aux besoins réels, désormais rendus solvables; de l'épargne, enfin protégée, aux besoins d'investissement des entreprises désormais conditionnés par la demande ; de l'emploi de la main d'œuvre, progressivement libérée, aux possibilités réelles de la technique productive et du marché » [Rougier L. (1938a), p. 85]. Cette conception de l'État repose sur un compromis qu'il convient de délimiter avec précision. Il faut un gouvernement fort, dans le cadre de ses attributions, pour être capable de résister aux intérêts coalisés, pour valoriser les richesses naturelles et les aptitudes individuelles. Il convient cependant de poser les limites à l'intervention de l'État par la reconnaissance des droits des citoyens, de tempérer le pouvoir exécutif par le contrôle législatif et le pouvoir judiciaire, de protéger les individus contre les abus de la puissance publique (ce sont les fameuses mystiques de l'économie dirigée, du marxisme et du régime corporatif des dictatures fascistes). Reste à trouver les moyens à mettre en œuvre pour instaurer les bases de ce « dirigisme juridique ». C'est ce que fera Louis Rougier en popularisant l'idée du libéralisme constructeur lors de la tenue du Colloque Lippmann ${ }^{19}$ et la mise en place du Centre International d'Études pour la Rénovation du Libéralisme (dont il sera le secrétaire général), à l'origine de la constitution de la Société du Mont Pèlerin (Denord, 2001 ; Margairaz, 2001, Steiner, 2007). 

institutions au sein du système libéral. Ses travaux tournent autour de son œuvre principale, l'Ordre social (1945), qui n'est pas sans rappeler l'Ordre de marché de Hayek, à deux exceptions près. D'une part, l'ordre social n'est jamais spontané, «il est toujours le produit de la coordination d'un certain nombre de comportements individuels » (Rueff, 1967, p. 84). Une coordination qui ne peut résulter elle-même que d'interactions s'exerçant entre les individus qu'elle unit. D'autre part, l'intervention de l'État ne doit pas être rejetée. Il existe des formes d'interventions de l'État qui sont non seulement nécessaires, mais également souhaitables (celles qui sont compatibles avec le libre jeu du mécanisme de prix).

D'une manière générale, l'ordre social renvoie à l'image d'une société pacifique et gouvernée. Pour parer à la fatalité de la guerre entre les hommes (utilisation de la force, de l'esclavage ou de la volonté du souverain dans l'état de nature), la société doit être pacifiée. Il suffit pour cela que «la jouissance et la disposition, qui par elles-mêmes, suscitent la convoitise des hommes, y soient rendues indésirables à toute personne autre que celle qui a été élue pour l'obtenir » (Rueff, 1948a, p. 507). L'imposition d'une paix sociale suppose ainsi l'application d'un système particulier de contrainte sociale: l'assujettissement de chaque chose à la volonté d'une seule personne. Ce droit exclusif de jouissance et de disposition d'une chose par une personne qualifie le droit de propriété, et c'est là, la première institution d'une société pacifiée. En soumettant toute chose à une personne, le droit de propriété divise l'univers en deux zones : celle du domaine possédé (intérieur) dans lequel l'individu est souverain, libre d'agir comme il le souhaite ; celle du reste du monde (domaine extérieur) dans lequel son action est contrainte par le consentement du propriétaire et des sanctions. Dans la droite lignée des jurisconsultes (Grotius, Pufendorf), Jacques Rueff rappelle que selon l'article 544 du Code civil, le droit de propriété est «le droit de jouir et de disposer d'une chose de la manière la plus absolue, pourvu qu'on n'en fasse pas un usage prohibé par les lois ou par les règlements " (Rueff, 1948a, p. 78). Le droit de propriété est donc la marque du monopole d'usage et de disposition accordé à une personne. Pour qu'il puisse remplir convenablement son rôle, il faut que son existence soit aisément prouvée à ceux qui viendraient le contester. Jacques Rueff précise cependant que le contenu du droit n'est pas la chose possédée, mais bien la faculté d'en jouir et d'en disposer. C'est cette faculté recherchée par les hommes, qui constitue la propriété et qui a pour eux de la valeur. Or la valeur est «cette qualité commune à toutes les richesses qui, par l'échange, peuvent remplir un même droit » (Rueff, 1948a, p. 80). On retrouve ici un argumentaire largement employé par Auguste Walras (1831) et Léon Walras (1874), le droit naturel et l'économie politique doivent s'éclairer mutuellement car la valeur et la propriété reposent sur les mêmes principes. Le droit de propriété apparaît ainsi « comme un véritable récipient à la valeur, de volume bien déterminé, susceptible d'être rempli, au gré de son titulaire et à concurrence de son volume, de toutes richesses offertes sur le marché, ou plus exactement de la valeur que représente la propriété de ces richesses » (ibid.).

Quatre modes d'appropriation des richesses sont susceptibles d'être présents dans une société : la violence, la dévolution légale, le don et l'échange. L'appropriation des richesses par l'échange constitue un fait général, permanent et universel, qui est du ressort de la science économique. Ainsi le transfert de propriété ne reposerait plus sur la violence mais bien sur le libre consentement de celui qui détient les richesses, donc sur l'échange. Jacques Rueff ajoute que dans les systèmes économiques complexes, il existe 
un intermédiaire commun à tous les échanges, la monnaie. En outre, pour que l'échange intervienne, «il faut évidemment qu'acheteurs et vendeurs soient mis en présence; le marché est l'endroit prévu et aménagé à cette fin» (Rueff, 1948a, p. 21. Ainsi prend naissance, le second acte de l'imposition de la paix sociale, la deuxième institution fondamentale : le marché. L'institutionnalisation du marché, en tant « qu'instrument d'un véritable suffrage universel dans le domaine économique » (Rueff, 1967, p. 255), s'effectue par l'intermédiaire du mécanisme des prix. Ce dernier joue un double rôle. D'une part, il permet de définir l'échange comme « un simple transfert du changement du contenu des droits de chacun des échangeurs, changement qui n'affecte pas les dits droits » (Rueff, 1948a, p. 96) et non plus comme un double transfert de droits de propriété. Ainsi tout titulaire de droits de propriété, désireux d'en modifier le contenu, n'a qu'à vider la richesse qui le constitue sur le marché où elle s'échange contre celle qu'il désire, et à remplir de celle-ci le droit ainsi libéré. Ainsi, grâce au mouvement de prix, «vidage et remplissage s'accomplissent simultanément, conformément au désir de tous les participants au marché » (Rueff, 1948a, p. 97). Le marché est dit en équilibre [Rueff J. (1948c)] et les échanges sont conservateurs de vrais droits. Lorsque les prix s'écartent des prix d'équilibre, ils seront générateurs de faux droits. D'autre part, il joue le rôle d'action contraignante destinée à garantir les droits du propriétaire et à modifier le comportement des individus qui ne sont pas propriétaires du bien en question. Le mécanisme des prix définit ainsi une procédure de récompenses et de sanctions propres au marché : « Pour tout vendeur, le prix est la récompense du sacrifice qu'il consent en se privant de l'objet venu; pour tout acheteur, il est le sacrifice consenti en vue de la satisfaction attendue de l'objet acquis » (Rueff, 1967, p. 205).

Si le mécanisme des prix est générateur de l'ordre collectif (Rueff, 1949), la liberté des prix «est la condition d'existence d'une monnaie saine" (Rueff, 1947). La monnaie constitue la $3^{\mathrm{e}}$ institution : "la monnaie de nos sociétés complexes est une création purement institutionnelle, dont les modalités peuvent varier à l'infini. Ce qui fait une monnaie, c'est son acceptation généralisée dans les échanges » (Rueff, 1957, p. 535). Cette institutionnalisation de la monnaie revêt plusieurs formes. La monnaie est tout d'abord vivante. Elle est liée à l'existence de créances : «la monnaie n'est pas la masse inerte qu'envisagent les théories mécanistes des phénomènes monétaires » (Rueff, 1957, p. 530). La monnaie reflète ensuite la souveraineté des utilisateurs de monnaie (la demande). S'opposant à la théorie keynésienne, Jacques Rueff précise que la monnaie en circulation sera dominée par un fait essentiel : « la liberté des utilisateurs d'encaisses de fixer à leur gré le montant de celle-ci» (Rueff, 1957, p. 532). La monnaie, en tant que richesse véritable (vraie créance), est enfin dotée par sa nature d'un certain pouvoir d'échange. Elle constitue à ce titre une condition d'existence du marché : c'est par le consentement tacite de tous les échangeurs du marché que cette richesse est « revêtue du caractère monétaire » (Rueff, 1957, p. 536).

Cela étant, une société pacifiée dans laquelle chaque chose est assujettie à la volonté d'une seule personne (dans laquelle le droit de propriété a été institutionnalisé) reste encore largement subordonnée aux sentiments nés spontanément dans le cœur des hommes (amour filial, horreur du meurtre, luxure, mensonge..) et aux volontés individuelles. Un monde où les fins sont imposées non par la collectivité des hommes mais par ceux dont le sort a pourvu de droits de propriété, ne peut rendre un pays heureux, puissant et prospère. Selon Jacques Rueff, «Pareil monde sera rejeté avec horreur par tous ceux qui pensent qu'une société d'hommes ne doit pas seulement 
donner à quelques-uns de ses membres les satisfactions que ceux-ci tiennent pour les plus désirables, mais doit tendre vers des fins qui la dominent, par tous ceux qu'anime une soif d'idéal et de progrès, de charité et de justice, de prospérité et de grandeur » (Rueff, 1948, p. 517). De tels hommes voudront que la société humaine soit gouvernée, tel un navire soumis à un pilote, vers les buts qu'ils lui assignent.

La société gouvernée doit ainsi se substituer à la société pacifiée, et nous entrons là dans la quatrième institution de l'ordre social, le gouvernement. Ce dernier, qu'il soit théocratique, moral ou civil, n'est que « l'art de modifier, par voie de contrainte, la destination donnée à certaines richesses, en substituant des fins collectives, sociales ou morales aux fins individuelles que leurs maîtres leur eussent imposées dans leur souveraineté de propriétaire " (Rueff, 1948, p. 527). Gouverner, c'est donc soustraire des richesses à la maîtrise de leurs propriétaires respectifs, pour les consacrer aux emplois que les autorités gouvernementales veulent pour elles. Deux méthodes sont alors envisageables. On peut chercher à déposséder le propriétaire d'un fragment de souveraineté dont on veut modifier l'usage et le transférer à l'autorité gouvernementale. L'impôt transfère au gouvernement les domaines de souveraineté qu'exige la mission gouvernementale. Jacques Rueff qualifie ce régime de gouvernement de libéral. On peut vouloir obtenir par voie de contrainte du titulaire du droit de propriété, qu'il choisisse lui-même, pour les richesses qu'il gouverne et dans sa pleine souveraineté, les fins que le gouvernement entend lui imposer. Contrairement à la première méthode qui réduit les champs de souveraineté individuels, la seconde méthode impose une modification des volontés qui engendrent les actes. La zone dans laquelle le titulaire de droits de propriété choisit librement est diminuée de celle qu'affectent les sanctions contraignantes. Jacques Rueff qualifie ce second régime de gouvernement d'autoritaire (ou socialiste). Si en théorie, le libéralisme et l'autoritarisme ne sont que deux méthodes de gouvernement qui présentent le caractère commun de soustraire à la souveraineté du propriétaire des fractions plus ou moins importantes de son domaine patrimonial, dans la pratique, Jacques Rueff note que les régimes gouvernementaux comportent toujours des interventions de deux ordres: "les régimes réels sont donc toujours, partie libéraux, partie autoritaires. Ils portent cependant l'un ou l'autre étiquette suivant le dosage des deux méthodes de gouvernement et suivant que, dans les cas où elles sont applicables, les autorités gouvernementales favorisent l'une ou l'autre d'entre elles» [Rueff J. (1948), p.533]. Il est ainsi possible, indépendamment de la méthode, de distinguer des programmes caractérisés par des dosages différents de l'intervention gouvernementale. Aux deux extrémités de ces programmes, on trouve l'individualisme, qui comme son nom l'indique, laisse aux individus la pleine souveraineté de leur patrimoine (ce régime ne comporte ni impôts, ni restrictions légales ou réglementaires aux droits de propriété), et le communisme, qui soustrait aux souverainetés individuelles la totalité de ces richesses (celles-ci deviennent la propriété des autorités sociales).

Le problème du gouvernement n'est donc au final qu'un problème de déplacement de certaines richesses par rapport à certaines volontés. Il peut être résolu par le déplacement de ces dites richesses (c'est la méthode libérale) ou par le déplacement des volontés (c'est la méthode autoritaire). Pour qu'une autorité gouvernementale ait un tel pouvoir, il faut et il suffit qu'elle réussisse à rendre indésirable tout acte accompli en violation des commandements qu'elle sanctionne de ses interventions. Mais également que tout individu qui n'aurait pas renoncé à un acte défendu, sache qu'il encourra un certain nombre de sanctions avant même son accomplissement : " pouvoir contraignant 
et volonté investie par lui sont les deux organes essentiels de l'appareil gouvernemental, ceux en dehors desquels aucun gouvernement ne pourrait exister »(Rueff, 1948a), p. 540). C'est là qu'intervient l'État. En tant que personne morale animée par des fins collectives et sociales, il incarne l'organe susceptible de traduire en actes les volontés dont il est l'instrument d'expression. Jacques Rueff précise qu'en fonction de la désirabilité de l'intervention publique et de l'indésirabilité des impôts, se trouvera fixé le volume des droits dont l'État dispose pour mener à bien sa mission gouvernementale: «La conscience publique veut-elle expansion et puissance: l'État remplira des services et capitaux susceptibles de les procurer les droits sur lesquels il exerce son action. Préfère-telle prospérité et expansion économique : il créera ou fera créer routes, ports, chemins de fer et canaux. Désire-t-elle le soulagement des misères imméritées ou l'atténuation des inégalités sociales : il attribuera, soit en nature, soit en monnaie, le contenu des droits qu'il commande aux personnes dont il veut améliorer le sort » (Rueff, 1948a, p. 544).

Dans le domaine économique, toute intervention de l'État se posera en termes d'opportunité de la dépense envisagée et d'absence de nuisance vis-à-vis du libre jeu du mécanisme des prix (Jacques Rueff oppose le régime des prix libres au régime des prix bloqués). Les interventions publiques ne sont donc limitées ni dans leurs aspirations collectives, ni dans leurs réalisations sociales, mais simplement par les ressources que l'État dispose. Les interventions incompatibles avec le libre jeu du mécanisme des prix portent sur les prix eux-mêmes. Il s'agit principalement de prix plancher (salaire minimum) ou de prix plafond (taux de l'usure, loyers), des taux d'intérêt, des taux de change. Les interventions nécessaires et souhaitables s'appuient sur le constat que la simple existence de l'institution "marché» n'assure pas son efficacité. Parmi les interventions qui ne portent pas atteinte au mécanisme des prix, il convient de dissocier celles qui agissent sur le cadre juridique et celles qui interviennent dans le cadre monétaire. Le système juridique est l'instrument fondamental de toute société civilisée et gouvernée. C'est par lui et par lui seul que l'autorité gouvernementale fait connaitre aux volontés individuelles l'état social qu'elle entend définir. Toutefois, l'édifice juridique sera constamment battu en brèche par les passions humaines (exemple des infractions fiscales qui doivent être durement réprimandées). C'est donc la réalité des droits et des obligations que l'État applique (la loi est à la fois synonyme d'obligations et de sanctions), qui donne une certaine existence à l'édifice juridique bâti par le pouvoir législatif: "sévérité des sanctions, rigueur dans leur application, efficacité dans la recherche des violations de droits de propriété et d'obligations fiscales, telles sont les conditions hors desquelles un ordre libéral ne saurait exister. Ceci montre combien est absurde la conception qui voit dans une société libérale un régime de relâchement, de désordre et de confusion » (Rueff, 1948a, p. 562).

Toutes les formes d'interventions institutionnelles ne sont donc pas équivalentes. Certaines sont nécessaires et préférables, d'autres sont au contraire préjudiciables. En donnant à la notion d'institution, une acceptation la plus large possible, «l'ensemble des règles de droit que l'organisme institué a mission de mettre en œuvre » (Rueff, 1958, p. 2), Jacques Rueff n'hésitera pas à présenter les 248 articles du Traité instituant la Communauté Économique Européenne comme un moyen de définir une fois pour toutes le marché commun. La notion de marché institutionnel illustre ainsi un fait important: celui d'attribuer à des institutions communautaires, le pouvoir de créer un marché: "c'est essentiellement parce qu'il crée non seulement un Marché commun mais un 
marché institutionnel que le Traité de Rome est différent de tous ceux qui antérieurement ont poursuivi vainement le même objet » (ibid.).

D'une certaine manière, on peut affirmer que la notion de marché institutionnel est l'aboutissement des années d'effort en vue de rénover la pensée libérale française. D'un point de vue théorique, le marché institutionnel délimite une zone géographique (le Marché commun) sur laquelle les comportements économiques des individus sont régis par le mécanisme des prix. Les producteurs et les consommateurs parviennent ainsi à atteindre le maximum de satisfaction pour un minimum de peine. Les offres et les demandes se rencontrent pour donner lieu à un équilibre. C'est le fameux ordre économique tant décrié par Jacques Rueff (1967). Le mécanisme des prix devient finalement "the instrument of economic democracy» (Rueff, 1948b, p. 539). D'un point de vue doctrinal, le marché institutionnel se distingue du marché manchestérien en ce sens que s'il est une zone de "laisser passer », il n'est pas une zone de «laisser faire ». Il ne s'agit pas de laisser le marché en proie aux intérêts privés (ententes tacites pour préserver certains débouchés) et aux résistances diverses (barrières douanières), mais bien de réencastrer le marché dans les structures politiques et sociales européennes: « Au laisser faire total, ils ont préféré un marché limité au domaine géographique dans lequel la création des institutions sans lesquelles le marché en pourrait ni exister, ni durer était politiquement possible » (Rueff, 1958, p. 3). Le marché institutionnel est donc à la fois un moyen de dépasser les limites du libéralisme du XIX siècle (foi dans l'émergence spontanée du marché) tout en réaffirmant les bienfaits de la liberté et un instrument de progrès social dans le sens où seule l'évolution des institutions permet aux hommes d'aspirer à une plus grande justice sociale. Ce libéralisme social ou socialisme libéral remet ainsi à leur place les querelles qui séparent libéraux et socialistes : «Le marché institutionnel doit ainsi rassembler les partis que préoccupe avant tout la liberté de la personne humaine et ceux qui, tout en refusant la contrainte des volontés individuelles, veulent, dans la répartition, moins d'inégalité et plus de justice. Libéraux et socialistes sont voués... aux disciplines du marché institutionnel. Assurément des nuances importantes les distingueront. Les uns voudront plus de liberté, les autres plus de satisfactions sociales. Mais leurs revendications s'exerceront dans le cadre d'une même structure : celle qu'établit le marché institutionnel » (Rueff, 1958, p. 5).

Du marché institutionnel à la planification concurrentielle, il n'y a qu'un pas à faire pour évoquer la position commune de Rueff et Allais : «la notion d'un marché institutionnel suivant la formule très heureuse de $\mathrm{M}$. Jacques Rueff, membre de la Cour de Justice de la CECA... me semble parfaitement définir le genre de compromis qui peut être établi entre deux tendances de doctrine à première vue tout à fait opposées. Elle correspond à la notion de planisme concurrentiel que j'ai moi-même suggérée depuis 15 ans comme d'une synthèse possible du libéralisme et du socialisme » (Allais, 1960, p. 177).

Là où peut être l'ingénieur économiste Maurice Allais semble aller plus loin, c'est lorsqu'il affirme que la réalisation de l'ordre structurel dans les pays sous-développés passe par une série d'interventions étatiques en cinq catégories : (1) l'action de l'État et la structure institutionnelle de l'économie de marché, « le mécanisme des prix ne peut fonctionner efficacement que dans le cadre d'une économie de marché institutionnelle et d'une planification des structures" (Allais, 1961a, p. 107); (2) l'action de l'État et l'infrastructure d'équipement (chemins de fer, routes, canaux, ports, postes et télécommunications); (3) l'action de l'État et l'éducation, «seul un haut niveau d'éducation générale et technique peut, d'une part fournir les élites économiques et 
politiques indispensables, et d'autre part donner à l'ensemble des citoyens le niveau de formation qu'exige un fonctionnement correct de la démocratie » (Allais, 1961a, p. 107); (4) l'action de l'État et la législation sociale, « la législation sociale ne peut et ne doit pas, au début, prendre modèle sur les législations avancées des pays développés, législations dont l'application serait trop coûteuse, compte tenu du bas niveau actuel de productivité et dont le seul effet serait de ralentir le développement » (Allais, 1961a, p. 109) ; l'action de l'État et la politique démographique, «le freinage de l'expansion démographique apparaît comme la condition première de tout développement économique réel » (ibid.).

Reste à conclure cette partie en revenant sur la position de Hayek. À travers le colloque Lippmann et les réunions de la Société du Mont Pèlerin, il est coutume de rappeler le dogmatisme de Hayek à toute intervention de l'État et sa défense de l'intérêt individuel. À la lecture de la Route de la Servitude, cette position nous semble plus nuancée. Si Hayek défend l'idée que le libéralisme est basé sur la conviction que la concurrence est le meilleur moyen de guider les efforts individuels, il ne nie pas que pour que la concurrence puisse jouer un rôle bienfaisant, « une armature juridique soigneusement conçue est nécessaire » (1946, p. 33). Si la concurrence en tant que principe d'organisation sociale exclut certains types d'interventions coercitives dans la vie économique, elle peut dans le même temps "exiger certains genres d'actions gouvernementales" (ibid.). Au final, Hayek est conscient que le fonctionnement de la concurrence requiert à la fois une articulation cohérente des différentes institutions que sont la monnaie, les marchés, l'information ainsi qu'un système juridique approprié (notamment concernant le droit de propriété) : «Il ne suffit nullement que la loi reconnaisse le principe de la propriété privée et de la liberté des contrats ; beaucoup de choses dépendent de la précision avec laquelle est défini le droit de propriété s'appliquant à des objets différents. L'étude systématique de la forme des institutions juridiques qui permettent un fonctionnement efficace de la concurrence a malheureusement été négligée ; il y a de ce côté de sérieuses défectuosités, en particulier en ce qui concerne les lois sur les sociétés et sur les brevets, qui non seulement ont entravé le jeu de la concurrence, mais encore l'ont détruite en bien des domaines » (1946, p. 34). Toutefois, admettre que la concurrence puisse accepter une certaine dose de réglementation, ne signifie pas qu'il faille se plier aux sirènes du planisme.

\section{Conclusion}

En cherchant à poser les bases d'un nouveau libéralisme, le courant néolibéral français, incarné par Louis Rougier, Jacques Rueff et Maurice Allais, a remis au goût des idées ancrées dans la tradition économique française (Say, Walras...) pour rejeter les thèses du laisser-fairisme et de la planification centralisée. Ainsi, la liberté n'est pas naturelle mais bien un droit que seule la société peut instituer ; la concurrence n'est pas spontanée, elle doit être organisée; des institutions correctement articulées (droit de propriété, monnaie, marché, État...) sont nécessaires au bon fonctionnement du système libéral ; le marché et l'État sont deux institutions interdépendantes... Face aux pseudo-vérités, le néolibéralisme français entend proposer une troisième voie réconciliant les principes $\mathrm{du}$ libéralisme et ceux du socialisme. Il s'agit ni plus ni moins que de redéfinir l'État « comme protecteur du cadre réglementaire et légal instituant le marché et comme administrateur des logiques concurrentielles censées y fonctionner »(Dardot, Laval, 2007, p. 114-115). 


\section{BIBLIOGRAPHIE}

Allais M. [1990], Louis Rougier. Prince de la Pensée, Les Terrasses de Lourmarin.

Allais M. [1989], La théorie des surplus, PUG, Grenoble.

Allais M. [1978], « Jacques Rueff 1896 - 1978 : la pensée et l'action », École Nationale Supérieure des Mines de Paris, réf. EMP 134.381, CCL 5162.

Allais M. [1970], Capitalisme et collectivisme, Le libéralisme, $3^{\mathrm{e}}$ séminaire de la pensée libérale, novembre, p. 93 - 107.

Allais M. [1968], « La signification économique et sociale de l'économie de marchés », Archives Rougier, Château de Lourmarin, R 11, Document $\mathrm{n}^{\circ} 2259$, version définitive du 5 décembre, $41 \mathrm{p}$.

Allais M. [1967], Manifeste pour une Société Libre, Centre d'Analyse Économique, 72 p.

Allais M. [1965], « Sur la planification », Cujas, réf. EMP 134.419, CCL 1447.

Allais M. [1964b], « La théorie économique et la tarification optimum de l'usage des infrastructures de transport ", Groupe de recherches économiques et sociales, ENSMP, 134.282 CCL 5162.

Allais M. [1961a], « Le tiers Monde au carrefour : centralisation autoritaire ou planification concurrentielle », Les Cahiers Africains, $n^{\circ} 7$ (152 p.) et n 8 (147 p.), Éditeurs Création de Press.

Allais M. [1961b], La politique de l'Occident à l'égard du Tiers Monde, Sirey, Paris.

Allais M. [1959], L’Europe Unie. Route de la Prospérité, Calmann-Lévy, Paris.

Allais M. [1950a], « Au-delà du laisser-fairisme et du totalitarisme », Nouvelle Revue d'économie Contemporaine, avril, p. 25-32.

Allais M. [1950b], « Au-delà du laisser-fairisme et du totalitarisme », Nouvelle Revue d'économie contemporaine, mai, p. 26-32.

Allais M. [1950c], « La démocratie et le totalitarisme », École Nationale Supérieure des Mines de Paris, Leçon 50, Document 503, p. 569-585. Texte publié dans la Revue d'économie contemporaine, novembre - décembre, p. 8-17, janvier 1951, p. 7-17.

Allais M. [1950d], L'économie soviétique est-elle efficiente ? Librairie de Médicis, Paris.

Allais M. [1949b] «Concurrentialisme et marxisme », Groupe de recherches économiques et sociales, Paris, ENSMP, le 20 avril, 16 p.

Allais M. [1949c], « Socialisme et libéralisme », $15^{\mathrm{e}}$ Congrès National de la Paix, 7-10 novembre, $6 \mathrm{p}$. Réimpression dans les Cahiers socialistes, Bruxelles, mai, p. 31-37.

Allais M. [1947a], « Note sur notre terminologie économique », Groupe de recherches économiques et sociales, Paris, ENSMP, le 21 janvier, $3 \mathrm{p}$.

Allais M. [1947b], Économie et Intérêt, Imprimerie Nationale, Paris.

Allais M. [1947d], Coordination des transports, le problème de la coordination des transports et la théorie économique, ENSMP, réf. 71.709 CCL 1121, 32 p.

Allais M. [1947e], « Pour un socialisme concurrentiel », La Fédération, septembre, 12 p. 
Allais M. [1946a], Abondance ou Misère, Librairie de Médicis, Paris.

Allais M. [1946c], « Deux conditions fondamentales d'un jeu efficace de la concurrence, la suppression du laisser-faire et la stabilité monétaire », Bulletin des transports et du commerce, août, $2 \mathrm{p}$.

Allais M. [1945a], Économie pure et rendement social, Sirey, Paris.

Allais M. [1945b], Prolégomènes à la reconstruction économique du monde, Sirey, Paris.

Allais M. [1943], À la recherche d'une discipline économique. Première partie, L'Économie Pure. $1^{\text {re }}$

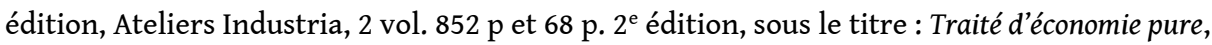

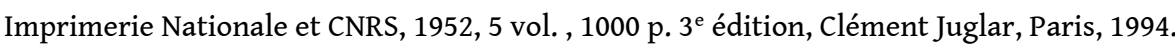

Arena R. [2000], « Les économistes français en 1950 », Revue économique, vol. 51, n 5, p. 969-1007.

Aron R. [1937], « Réflexions sur les problèmes économiques français », Revue de Métaphysique et de Morale, $\mathrm{n}^{\circ}$ 4, p. 793-822.

Audier S. [2008], Le colloque Lippmann : aux origines du néolibéralisme, Le Bord de l'Eau, Lormont.

Berndt C., Marion M. [2006], « Vie et œuvre d'un rationaliste engagé : Louis Rougier (1889 1982) », Philosophia Scientiae, vol. 10, (2), p. 11-90.

Bilger F. [2003], « La pensée néo-libérale française et l'ordo-libéralisme allemand » in P. Commun (dir), L'Ordolibéralisme allemand, aux sources de l'économie sociale de marché, CIRAC.

Brunelle D. [2008], « Serge Audier, le colloque Lippmann : aux origines du néolibéralisme », l' Actualité Économique, vol. 84, n² 2, p. 235-237.

Casanova J.C [1986], « Des anciens aux modernes, les raisons du renouveau libéral », Conférence sur le concept de liberté au Wilson Center de Washington, Commentaire, $\mathrm{n}^{\circ}$ 35, p. 509-518.

Classens E. [1967], Les fondements philosophiques des systèmes économiques, Payot, Paris.

Claves F.U [2004], « Walter Lippmann et le néolibéralisme de la Cité libre », Cahiers d'économie politique, $\mathrm{n}^{\circ} 48$, p. 79-110.

Cobden R. [1835], England, Irland and America, by a Manchester Manufacturer, William Tait, Edinburgh.

Collectif [1961], « Les tendances modernes du libéralisme économique », Les Essais, n 11-12.

Cros J. [1951], Le néo-libéralisme. Étude positive et critique, Librairie de Médicis, Paris.

Dard O. [2000], « Théoriciens et praticiens de l'économie : un changement de paradigme », in S. Berstein et P. Milza (dir.), L'année 1947, Presses de Sciences-Po, Paris, p. 75-114.

Dardot P., Laval C. [2007], « La nature du néolibéralisme : un enjeu théorique et politique pour la gauche ", Mouvements, $n^{\circ}$ 50, p. 108-117.

Dayre J. [1959], Les faux dilemmes : libéralisme ou dirigisme ? Inflation ou récession, Paris, Éditions d'Organisation, $301 \mathrm{p}$.

Denord F. [2008], « Néo-libéralisme et économie sociale de marché. Les origines intellectuelles de la politique européenne de la concurrence (1930 - 1950) », Histoire, Economie et Société, $27^{\mathrm{e}}$ année, p. 23-33.

Denord F. [2007], « Le prophète du néolibéralisme français », Philosophia Scientiae, vol 7, p. 121-133. 
Denord F. [2007], Le néo-libéralisme version française, histoire d'une idéologie politique, Démopolis, Paris.

Denord F. [2004], « La conversion au libéralisme », Mouvements, n 35, sept.-oct., p. 17-23.

Denord F. [2003], « Genèse et institutionnalisation du néolibéralisme en France (1930 - 1950) », Thèse de doctorat de sociologie, EHESS.

Denord F. [2002], « Le prophète, le pèlerin et le missionnaire : la circulation internationale du néo-libéralisme et ses acteurs ", Actes de la recherche en Sciences Sociales, vol 5, n 145, p. 9-20.

Denord F. [2001], « Aux origines du néo-libéralisme en France Louis Rougier et le Colloque Walter Lippman de 1938 ", Le Mouvement Social, n 195, avril-juin, p. 9-34.

Detoeuf A. [1938], Construction du syndicalisme, Gallimard, Paris.

Diemer A., Laperche B. (2014), De la critique des corporations à la libéralisation des forces productives : l'économie politique de Jean Baptiste Say ", Cahiers d'économie et d'innovation, $p$. 19-38

Diemer A. [2013b], « Jacques Rueff, de la crise de 1929 à l'ordre social et au marché institutionnel », Journée Rueff, IPAG, 23 mai, 17 p.

Diemer A. [2013a], « Louis Rougier, un économiste au service du renouveau du libéralisme international », Document de travail n 5, Université Blaise Pascal, 17 p.

Diemer A. [2012], « Aspects théorique et pratique de la concurrence dans l'œuvre de Maurice Allais ", Cahiers d'économie politique, vol. 62, p. 75-116.

Diemer A. [2011], « Institutions et institutionnalisation du courant néolibéral français : de Louis Rougier à Maurice Allais ", Séminaire du Greqam, Aix Marseille, 11 mars, 26 p.

Diemer A. [2010], « La planification concurrentielle, théorie et applications », in Diemer A., Lallement J., Munier B. (éd.), Maurice Allais et la Science économique, Clément Juglar, p. 189-213.

Diemer A. [2010], « La place des institutions dans le courant néo-libéral français : de Jacques Rueff et Louis Rougier à Maurice Allais », XIII ${ }^{\mathrm{e}}$ colloque ACGPE, Les Institutions dans la pensée économique, Paris, 27-29 septembre, $19 \mathrm{p}$.

Diemer A., Guillemin H. [2009], « La marchandisation du travail dans la société anglaise de la fin du XVII ${ }^{\mathrm{e}}$ siècle au début du XIX ${ }^{\mathrm{e}}$ siècle ", Cahiers d'Histoire, $\mathrm{n}^{\circ}$ 110, octobre-décembre, p. 53-82.

Diemer A., Lallement J. (2004), « De Auguste à Léon Walras : retour sur les origines du marché et de la concurrence walrassiennes ", Colloque de l'Association internationale Walras, Nice, 22-24 septembre, $23 \mathrm{p}$.

Engel P. [2007], « Vies parallèles : Rougier et Cavaillès », Philosophia Scientiae, vol. 7, p. 19-48.

Fridenson P. [2009], « Le Colloque Lippman, aux origines du néolibéralisme », Le Mouvement Social, $\mathrm{n}^{\circ} 227$, avril-juin, p. 138-140.

Frobert L. [2010], « Conventionalism and Liberalism in Jacques Rueff's Early Works : 1922-1929 », The European Journal of the History of Economic Though, vol. 17, p. 439-470.

Hawtrey R.G [1946], «L'Ordre Social, tomes I et II by Jacques Rueff », Economica, vol. 13, n 52, novembre, p. $300-303$.

Hayek F. [1946], La route de la servitude, Librairie de Médicis, Paris.

Lane G. [1996], « Jacques Rueff, un libéral perdu chez les planistes », Institut Euro 92, mars, 23 p. 
Lange O. [1949], « The Practice of Economic Planning and The Optimum Allocation of Resources », Econometrica, vol. 17, Report of the Washington Meeting, juillet, p. 166-171.

Lecoq T. [1989], « Louis Rougier et le néolibéralisme de l'entre deux guerres », Revue de Synthèse, avril-juin, p. 241-255.

Leroyer S. [2003], « Devoirs et libéralisme, vers un renouveau du contrat social ? ", CRDF, n², p. $125-138$.

Lippmann W. [1937], The Good Society, Little Brown and Company Edition, traduction française, La Cité libre, Librairie de Médicis, 1938.

Lhoste-Lachaume P. [1936], Réalisme et sérénité, synthèse pratique de pensée et d'action, Alcan, paris.

Lhoste-Lachaume P. [1950], Réhabilitation du libéralisme, t. 1, SEDIF, Paris.

Lhoste-Lachaume P., Audouin R. [1962], Le Corporatisme, pseudo remède contre l'étatisme, SEDIF, Paris.

Margairaz M. [2001], « Entre Néo-libéralisme et corporatisme : apports et limites de deux trajectoires singulières ", Le Mouvement Social, $n^{\circ}$ 195, avril-juin, p. 3-7.

Margairaz M. [1991], L'État, les finances et l'économie : histoire d'une conversion 1932-1952, Comité pour l'histoire économique et financière de la France, Paris.

Maurois A. [1938], Préface à la Cité libre de Walter Lippmann, Librairie de Médicis, Paris.

Michel J. [2008], « Peut-on parler d'un tournant néo-libéral en France ? », International Web Journal, vol. 5, p. 1-17.

Myrdal G. [1960], Beyond the Welfare State : Economic Planning and Its International Implications, Yale University Press, traduction française, Planifier pour développer, Éditions ouvrières, Paris, 1963.

Nadeau R. [2007], « Le conflit des libéralismes », Philosophia Scientiae, vol. 7, p. 134.

Perroux F., Tinbergen J., Rueff J., Domar E.D, Lundberg E.F, Kalecki M., Zagorski J., Dalal K. [1949], "The Practice of Economic Planning and the Optimum Allocation of Resources : Discussion », Econometrica, vol. 17, juillet, p. 172-178.

Perrot E. [2005], « Néo-libéralisme », Études, vol. 2, p. 1-10.

Piatier A. [1938], « Ludwig von Mises, Le socialisme ; Louis Rougier, Les Mystiques économiques ;

Walter Lippmann, La Cité libre ; Maurice Bouvier-Azam, La Doctrine Corporative », Politique étrangère , vol. 3, n 6, p. 634-639.

Pirou G. [1939], Néo-libéralisme, néo-corporatisme, néo-socialisme, Gallimard, Paris.

Polanyi K. [1944], La Grande transformation, traduit de l'anglais par C. Malamoud et M. Angeno, Gallimard, Paris, 2009.

Pont J.C (2006], « Avant propos », Philosophia Scientiae, vol 10, (2), p. 2 - 4.

Reybaud L. [1860], « Richard Cobden et l'école de Manchester », Revue des Deux Mondes, t. 27, 15 mai, p. 257-312.

Robbins L. [1938], Economie planifiée et ordre international, Editions Médicis.

Rougier L. [1959], « Scandale à Polytechnique » in M. Allais, Un Savant Méconnu, Clément Juglar, p. 55-75.

Rougier L. [1958], « L'État dirigiste », Écrits de Paris, février, p. 116-126. 
Rougier L. [1952], « Le premier gouvernement totalitaire de l'Europe », Écrits de Paris, février, p. 66-77.

Rougier L. [1949], « Le patriotisme idéologique », Écrits de Paris, août, p. 86-96.

Rougier L. [1948], « La logique de l'alternative et l'avènement des tyrannies », Écrits de Paris, février, p. 73-81.

Rougier L. [1939], Le Colloque Walter Lippman, Librairie de Médicis, Paris.

Rougier L. [1938a], Les Mystiques économiques, Librairie de Médicis, Paris.

Rougier L. [1938b], » Retour au libéralisme », Revue de Paris, $1^{\mathrm{er}}$ janvier, p. 62-81.

Rougier L. [1935], Les Mystiques Politiques Contemporaines, Sirey, Paris.

Rougier L. [1934], La Mystique soviétique, Équilibres, Bruxelles.

Rougier L. [1929], La Mystique démocratique, Flammarion, Paris.

Rueff J. [1979], « Politique économique », CEuvres complètes, t. III, Plon, Paris.

Rueff J. [1977], « De l'aube au crépuscule, Autobiographie », Euvres complètes, t. I, Plon, Paris.

Rueff J. [1969], Des Sciences physiques aux sciences morales. Un essai de 1922 reconsidéré en 1969, Payot, Paris.

Rueff J. [1968], « Remise à Maurice Allais du Grand Prix André Arnoux : discours de Jacques Rueff, Président du Jury » in M. Allais, Un Savant Méconnu, Clément Juglar, p. 77-92.

Rueff J. [1964], « Facteurs et mécanismes déterminant la quantité de monnaie en circulation », Cahiers du Séminaire d'Économétrie, $\mathrm{n}^{\circ}$ 7, La monnaie, p. 7 - 21.

Rueff J. [1958], « Une mutation dans les structures politiques : le marché institutionnel des communautés européennes ", Le Monde économique et financier, 9-10 février, p. 5.

Rueff J. [1955], « Un instrument d'analyse économique : la théorie des vrais et des faux droits », Conférence prononcée au Collège libre des sciences sociales et économiques, in Claassen E.M (dir.), Les Fondements philosophiques des systèmes économiques, Payot, Paris, 1967.

Rueff J. [1949], « Sur la théorie quantitative et le phénomène de régulation monétaire », Econometrica, vol. 17, Juillet, p. 295-306.

Rueff J. [1948a], L'Ordre social, $2^{\text {nd }}$ éd. (1e éd. 1945), Librairie de Médicis, Paris.

Rueff J. [1948b], « The Case of the Free Market », Foreign Affairs, vol. 26, n 3, p. $528-541$.

Rueff J. [1948c], « Le concept d'équilibre en économie », Congrès des économistes de langue française, Rapport de M. Dupriez, Domat-Montchrestien, Paris.

Rueff J. [1947], « The Fallacies of Lord Keynes General Theory », The Quaterly Journal of Economics, vol 61, n³, May, p. $343-367$.

Rueff J. [1947], Monnaie saine ou monnaie totalitaire, SEDIF, Paris.

Rueff J. [1946], « Sur les fondements de l'ordre dans les sociétés humaines », Conférence prononcée le 25 mai 1946 à la Société Française de Philosophie, in Claassen E.M (éd.), 1967.

Rueff J. [1939], « Préface à l'ouvrage de Charles Taquey », Richard Cobden, Un révolutionnaire pacifique, Gallimard, Paris.

Rueff J. [1935], « La crise du capitalisme », Conférence prononcée le 5 janvier 1935 à l'Académie des Sciences Morales et Politiques, in Claassen E.M (éd.). 
Salin P. [1989], « La pensée économique de Jacques Rueff » in Bourricaud F., Salin P. (éd.).

Schumpeter J.A [1954), History of Economic Analysis, Oxford University Press, traduction française, Histoire de l'analyse économique, 3 volumes, Editions Gallimard, 1983.

Shonfield A. [1967], Le Capitalisme d'Aujourd'hui. Introduction de Pierre Massé, traduit de l'anglais par B. Cazes et G. Gefen, Gallimard, Paris.

Spencer H. [1850], Le droit d'ignorer l'État, traduit de l'anglais par M. Devaldès, Belles Lettres, Paris, 1993.

Steiner Y. [2007], « Louis Rougier et la Mont Pèlerin Society : une contribution en demi-teinte », Philosophia Scientiae, vol. 7, p. 65 - 99.

Steiner Y. [2006], «L'apport de l'ordolibéralisme au renouveau libéral, puis son éclipse », Carnets de bord, $\mathrm{n}^{\circ} 11$, septembre, p. 94-104.

Steiner Y. [2005], « Louis Rougier et la Mont Pèlerin Society : une contribution en demi-teinte », Cahiers d'épistémologie du département de philosophie, Université du Québec, n²005-10, p. 38-39.

Taquey C. [1939], Richard Cobden, Un révolutionnaire pacifique, Gallimard, Paris.

Triffin R. [1947] «L'Ordre social by Jacques Rueff », The American Economic Review, vol. 37, n 1, mars, p. 213-215.

Vallon L. [1939, 1961], « L’offensive du Néolibéralisme », Syndicats, 4 janvier.

Villey D. [1967], « Jacques Rueff, un libéral moderne », in E. Claassen (dir.), Les fondements philosophiques des systèmes économiques, Payot, Paris.

Walras L. [1909], « Ruchonnet et le socialisme scientifique », CEuvres Complètes, vol. VII, Mélanges d'économie politique et sociale, Économica, Paris, 1987.

Walras L [1874], Eléments d'économie politique pure, CEuvres Complètes, vol V, Economica.

\section{NOTES}

1. Louis Rougier est avant tout présenté comme l'un des pères de la philosophie analytique de langue française (Berndt, Marion, 2006). C'est lui qui organisa avec Carnap, Franck, Neurath et Reichenbach, le premier colloque international de la philosophie scientifique à la Sorbonne en septembre 1935. Il bénéficiait d'une certaine Aura auprès des maîtres à penser du Cercle de Vienne (Pont, 2006, p. 3). Sa mauvaise réputation (son nom est associé à l'extrême droite, on l'accuse d'avoir été un collaborateur sous l'Occupation et d'être un antisémite) semble avoir joué un rôle non négligeable dans l'étude de son œuvre.

2. Son grand-père Paul Rougier (1826-1901) était professeur d'économie politique à la Faculté de Lyon et fondateur de la Société d'Économie Politique (1866) et d'Économie Sociale de Lyon (1889). Il en sera le secrétaire général pendant plusieurs années (Engel, 2007).

3. Dans la préface de la Cité Libre, André Maurois (1938, p. 3) note qu'avec « le livre de Walter Lippmann, avec celui de Louis Rougier sur les Mystiques économiques, avec celui du professeur viennois Ludwig von Mises sur le Socialisme, nous assistons, en ces trois pays différents, à une renaissance intellectuelle du libéralisme ».

4. Louis Rougier note que Walter Lippmann examine «ces mouvements sociaux non seulement sous leur forme fasciste et communiste, mais aussi dans le collectivisme progressif des États démocratiques, en essayant de déterminer si une société peut être planifiée et dirigée pour vivre dans l'abondance et en paix » (Rougier, 1938a, p. 11). 
5. Certains proposèrent de ne plus faire référence au libéralisme afin d'éviter certaines connotations. Louis Baudin Auguste Detoeuf, Jean-Louis Marlio considéraient que le mot libéralisme renvoyait directement aux travaux des disciples de Bastiat, Guyot ou Molinari. Ces économistes manchestériens qui avaient prôné le laisser-faire, le laisser-passer. Louis Baudin entendait lui substituer le mot individualisme qui aurait l'avantage de placer le colloque sous la bannière des grands classiques, Adam Smith, Jean-Baptiste Say, Stuart Mill... De son côté, Robert Marjolin soulignait que le mot liberté prêtait à confusion. Dans un contexte d'agression extérieure, il était judicieux de renoncer à certaines libertés pour éviter la guerre. D'autres tels que Louis Rougier et Jacques Rueff considéraient que le mot libéralisme était le bon mot. Il impliquait le respect d'un ordre légal qui permettait aux individus de vivre ensemble. Ce sentiment semblait partager par Ludwig von Mises, Jean Louis Marlio et José Castillejo pour qui le libéralisme est l'unique rempart aux idées totalitaires (son abandon serait interprété comme un recul, sinon une concession au collectivisme) et synonyme de libération face à l'absolutisme.

6. Selon Berndt et Marion [(2006), p. 22], Louis Rougier serait venu au libéralisme : « en précisant un point de vue à l'origine foncièrement anti-égalitaire, donc anti-communiste, pour lequel la démocratie est avant tout un nivellement par le bas ».

7. Ludovic Frobert (2010), p. 465) note que « Rueff could become one of the earlier representatives and then one of the leading figures of french neoliberalism, a movement that was to take form in 1935 to 1940 period".

8. La mystique est présente dans un grand nombre d'ouvrages de Rougier: la Mystique démocratique (1929), la Mystique soviétique (1934), les Mystiques politiques contemporaines (1935), Les Mystiques économiques (1938).

9. François Denord (2007), p. 121) note que le choix du terme «libéralisme constructeur » fait sans doute référence au « socialisme constructif » d'Henri de Man, théoricien du planisme (Man, 1933). Par la suite, Louis Rougier a abandonné cette dénomination pour celle « d'économie du consommateur » (Nadeau, 2007). Ce régime est basé sur «le plébiscite des prix sur les marchés concurrentiels, par le moyen desquels les consommateurs font connaître l'ordre de priorité et d'urgence de leurs besoins » (Rougier, 1949, p. 220, cité par Nadeau, 2007, p. 137).

10. Louis Rougier précise que «l'expérience, en France, du Front populaire montre pareillement comment, avec les meilleures intentions du monde, on peut s'orienter vers un régime totalitaire, dans le cadre constitutionnel d'une démocratie libérale, par une politique sociale qui impose des charges telles à la production qu'elle ne peut plus supporter la concurrence des prix mondiaux et doit se développer en vase clos; par des dépenses démagogiques auxquelles le trésor public ne peut pouvoir que par un appel autoritaire aux capitaux privés ou aux manipulations monétaires qui ruinent la capacité d'achat des consommateurs et ébranlent le crédit de l'État » (1938a, p. 21). 11. À ce stade, on peut considérer à travers la correspondance Röpke - Rougier et Röpke - Rueff que les thèses défendues par les néolibéraux français sont très proches de celles portées par les ordolibéraux allemands (voir archives Rougier, Château de Lourmarin).

12. «L'appareil des droits légaux dans toute société existante est un domaine de la plus grande complexité, le résultat acquis par des siècles de législation et de décisions judiciaires. Déterminer en quoi ces droits devront consister, pour satisfaire le public dans son choix délimiter leur portée et leur contenu, voilà une tâche de la plus haute difficulté. À quels objets s'étendront les droits de propriété ? S'appliqueront-ils aux idées et aux inventions ? Ou bien seront-ils limités à de rares ressources matérielles et à leur utilisation?» (1938, p. 204).

13. Dans le Traité d'économie pure (1943, 1952, 1994), Maurice Allais précise que l'ouvrage de Robbins a fait partie de ses premières lectures.

14. On ne peut pas à proprement parler, associer Walras au libéralisme, sa position est plus nuancée (libéral social) toutefois les rénovateurs du libéralisme que sont Rougier, Rueff et Allais font régulièrement référence à Léon Walras, l'un des pères de l'équilibre général. 
15. «Cela fait, il resterait à vider une seconde question sociale, celle de la production de la richesse par les hommes en société, en empêchant certains entrepreneurs de faire des bénéfices autrement que dans les conditions normales de la libre concurrence, c'est-à-dire par leur coup d'œil, leur habileté, leur économie. On y arriverait en faisant intervenir l'État soit pour exercer les entreprises lui-même, soit pour les concéder au mieux de l'intérêt social, toutes les fois qu'elles ne sont pas susceptibles de libre concurrence. C'est cette seconde question sociale qui faisait l'objet de mon cours d'économie politique appliquée [541, comme théorie appliquée naturelle à élaborer au point de vue de l'utilité, et qui fait celui de mes Études d'économie politique appliquée. Dire libre concurrence n'est pas du tout, comme on voit, dire absence de toute intervention de l'État. Et, d'abord, cette intervention est nécessaire pour établir et maintenir la libre concurrence là où elle est possible. La tendance des propriétaires fonciers, travailleurs et capitalistes est de constituer le monopole des services ; celle des entrepreneurs est de constituer le monopole des produits. Si ce monopole est contraire à l'intérêt public, l'État doit l'empêcher toutes les fois qu'il n'est pas fondé sur le droit naturel » (Walras, vol X, p. 425-426).

16. Cette référence à Hume n'est pas anodine, même chez Walras (Walker, 2004).

17. Louis Rougier fut proche de Röpke au point de publier dans la revue allemande Ordo (Rougier, 1962).

18. Louis Rougier revint sur le statut de la société anonyme en précisant que pour subsister l'économie libérale doit réviser l'ordre juridique existant. La société anonyme (loi de 1867) est une création de la législation qui lui confère le double privilège de la limitation des responsabilités (en fonction des apports) et de la transmission perpétuelle (cession des actions). Or cette innovation juridique, comme le rappelle Walter Lippmann (1938), p. 26) a été à l'origine de la concentration industrielle, verticale et horizontale, donnant naissance à des unités capitalistiques telles que les trusts. Parmi les réformes à apporter aux sociétés anonymes, Louis Rougier propose « d'interdire aux administrateurs de retenir les bénéfices au-delà de ce qui est nécessaire pour l'amortissement et les réserves de roulement et d'en faire des réinvestissements sans les soumettre à l'épreuve du marché des capitaux régi par la concurrence » (Rougier, 1938, p. 82).

19. Mais également du Colloque Paul Van Zeeland (1939) et du Colloque Sir William Beveridge (1940).

\section{RÉSUMÉS}

Au lendemain de la crise de 1929 et de la remise en cause des idées libérales, un certain nombre d'économistes (Lippmann, Röpke, Rougier, Rueff, Allais...) vont chercher à poser les bases d'une refondation du libéralisme. Ce mouvement n'est pas homogène, cependant le cœur de sa doctrine s'articule autour des deux positions suivantes: (i) dénoncer la montée du collectivisme et du planisme; (ii) rejeter les thèses défendues par les tenants du laisser-fairisme. Le colloque Lippmann constitue la première pierre de cette reconstruction, il se présente également comme une tentative visant à institutionnaliser le libéralisme dans l'histoire des faits et des idées. En France, Louis Rougier, Jacques Rueff et Maurice Allais incarnent ce processus de refondation du libéralisme. Tous trois défendent l'idée que ce nouveau libéralisme suppose une ingérence de l'Etat. Le libéralisme aurait ainsi besoin d'institutions solides pour conforter ses bases et asseoir sa légitimité. 
In the aftermath of the 1929's crisis and the questioning of liberal ideas, some economists (Lippmann, Röpke, Rougier, Rueff, Allais ...) tried to lay down the groundwork for an overhaul of liberalism. That movement is not homogeneous. However the heart of its doctrine revolves around two positions: (i) denounce the rise of collectivism and planning; (ii) reject the arguments of «laissez-fairisme ». The symposium Lippmann is the cornerstone of that reconstruction, it also presents itself as an attempt to institutionalize liberalism in the history of facts and ideas. In France, Louis Rougier, Jacques Rueff and Maurice Allais embody that process of liberalism's recasting. All three support the idea that "neoliberalism» requires State's interference. Liberalism needs strong institutions to consolidate its bases and its legitimacy

\section{INDEX}

Mots-clés : État, institutions, marché, planification, propriété

Keywords : institutions, market, planning, property, State

Code JEL B20 - General, B30 - General, B50 - General, K11 - Property Law, N01 - Development of the Discipline: Historiographical; Sources and Methods, P11 - Planning; Coordination; and Reform

\section{AUTEUR}

\section{ARNAUD DIEMER}

Université Blaise Pascal, TRIANGLE (ENS Lyon) 